\title{
植物繁育系统研究的最新进展和评述
}

\author{
何亚平 ${ }^{12}$ 刘建全 ${ }^{*}$ \\ (1 中国科学院西北高原生物研究所,西宁 810001) \\ (2 中国科学院研究生院 北京 100039)
}

摘 要 植物繁育系统是当今进化生物学研究中最为活跃的领域。繁育系统是指代表所有影响后代遗传组成的 有性特征的总和，主要包括花形态特征、花的开放式样、花各部位的寿命、传粉者种类和频率、自交亲和程度和交配 系统 其中交配系统是核心。我们重点综述了植物有性繁育系统研究中 1)传粉模式的多样性 ,2) 从历史发生角度 利用系统发育方法检验花性状的演化和繁育系统的生态转变过程 3)近交衰退对植物生殖史的影响及其机制和 4) 混和交配系统的时空动态、维持机理以及进化趋势的最新研究结果。总结了繁育系统在研究花适应性、物种形成 机制和濒危植物保护生物学中的重要作用。最后对今后的繁育系统研究提出了两点建议:1) 正确理解和使用适应 概念, 更多地利用人工控制实验来检验花的适应价值和 2) 将分子技术和生态学结合起来, 开展群落水平和种以上 水平的繁育系统研究, 比较驱动与传粉多样性密切相关的花多样性基因和自花、异花传粉基因的进化与分化, 确定 繁育系统相互作用中的关键因子。

关键词 繁育系统 进化 系统发育 多样性

\section{A REVIEW ON RECENT ADVANCES IN THE STUDIES OF PLANT BREEDING SYSTEM}

\author{
HE Ya-Ping $^{1}{ }^{2}$ and LIU Jian-Quan ${ }^{1 *}$ \\ (1 Northwest Plateau Institute of Biology, the Chinese Academy of Sciences , Xining 810001, China) \\ (2 Graduate School, the Chinese Academy of Sciences , Beijing 100039, China)
}

\begin{abstract}
The plant breeding system has become the most active and promising area among the recent evolutionary studies. In its definition, the plant breeding system represents the sum of all sexual characteristics that could possibly influence the genetic composition of subsequent generations, mainly encompassing floral morphological features, display patterns, longevity , species and visitation rates of pollinators , degree of self-compatibility and mating system. Among them the mating system is the most critical. In this paper we presented a review of the recent advances in the studies of plant breeding systems, with an emphasis on 1) the diversity of pollination in floral morphology, sexual expression , floral longevity and pollinator ,2) testing the evolution of the sexual characters and ecological shifts of the breeding system , such as sex expression , selfing and outcrossing in historical context by an independent phylogenetic approach ,3) effects of inbreeding depression on the plant reproduction cycle and their possible mechanisms , and 4) spatial and dynamic patterns of the mixed mating system and their maintenance mechanisms and evolutionary trends. We further summarized the application of plant breeding system research in the studies of flower adaptation , speciation and conservation of endangered species. Lastly , for future research, we suggested that 1) the word" adaptation" should be less used in flower adaptation explanations and more valuations should be based on the results of manipulated experiments and 2) both molecular and ecological methods should be combined to elucidate the plant breeding system at the scale of the community and high taxonomic levels, to compare the evolution and differentiation of the genes which drive flower diversity and control differentiation of the inbreeding and outcrossing , and to find the determinative factors among the interactive relationships in the breeding system.
\end{abstract}

Key words Breeding system, Evolution, Phylogeny , Diversity

被子植物生活史就是植株 - 受精卵 - 植株的循 环, 但决不是物理的重复, 前一过程经历传粉和生
殖 其实质是基因型选择，包括传粉、雌雄配子体选 择; 后一过程经历发育, 植株形态建成, 实质是表现 
型选择。生活史中, 生殖和发育都是表现型和基因 型间实现生物学转变的必经之路, 生殖和生殖前传 粉的研究内容就属繁育系统的研究范畴, 同时它还 包括传粉者的行为研究 (Holsinger,2000)。更为广义 的植物繁育系统还包括繁殖过程中不存在性表达的 克隆繁殖, 该领域的研究进展已有专门评述 (董鸣, 1996）。繁育系统通常的定义是代表直接影响后代 遗传组成的所有有性特征 (Wyatt, 1983)，主要包括花 综合特征、花各性器官的寿命、花开放式样、自交亲 和程度和交配系统, 它们结合传粉者和传粉行为是 影响生殖后代遗传组成和适合度的主要因素, 其中 交配系统是核心。尽管繁育系统中诸如花的开放式 样、花的形态特征及花各性器官的寿命等因素也严 重影响植物的交配系统，但交配系统(周世良等， 1998) 在定义上严格地说是指居群个体之间的交配 式样, 即自交和近交状态的总和, 也就是谁和谁交配 及其频率 (Barrett \& Harder，1996) 现代传粉生物学 的工作中, 交配系统的定量测定已经成为繁育系统 研究中相对独立的必需内容, 理论、方法和实践的研 究可参见(张大勇等,2001; 何田华等,2001; 汪小凡 等, 2001 陈小勇等, 2001 张冬梅等, 2001)。

繁育系统的研究始于人类文明之初。大约在公 元前 1500 年,亚术王宫就有对㫫楖树 (Phoenix dactylifera) 进行人工授粉的浮雕 ( Faegri \& van der Piji 1979; Proctor et al., 1996 ; 黄双全等,2000)。18 世纪初, Dobbs 和 Müller 首先发现植物异交现象以及 昆虫在植物异交中的作用 (Faegri \& van der Piji, 1979 ; Proctor et al ., 1996)。18 世纪下半叶, Kolreuter 发明人工杂交, Sprengel 创立了花理论 (花为昆 虫传粉而设计), 这两项工作是研究繁育系统的最基 本的方法 (Real ,1983)。Darwin 利用人工杂交方法揭 示了自交和异交的效果及不同花型对繁育系统的适 应和影响 建立起了植物繁育系统研究的基本理论 和方法 ( Darwin, 1862; 1876; 1877 ;Real , 1983 ; Proctor et al ., 1996 ; Richards , 1997)。达尔文开创的植物 繁育系统研究距今已有 140 多年, 该学科的发展曾 在相当长的一段时间内未受到人们应有的重视 (Lloyd \& Barrett ,1996)。近年来, 由于人们对生物多 样性不断减少的担心，转而关注生态系统中动植物 的相互关系, 重新重视植物繁育系统的研究 (Proctor et al . 1996) ,并取得了新的进展, 如野外观察中令 人惊奇的异花传粉机制不断被发现、野外实验观察 中得到的繁育系统多样性转变能得到分子系统发育 树进一步的检验、分子生物学领域中有关异花传粉
中花结构变异发育的分子控制过程和花粉-柱头识 别机理不断被深入。人们不再只是简单描述花外部 性状的性表达方式及其对昆虫的传粉适应, 而是将 花各性器官的表达和寿命、花的开放式样、远交衰 退、基因流、花发育模式、花粉-柱头相互识别等新概 念，以及定量测定和分析、定性模拟等方法引入到繁 育系统研究中来。交配系统的研究由于等位酶技 术、RAPD、SSR、AFLP 等分子标记技术在繁育系统中 的成功应用, 进入了一个新的研究层次; 同时一些原 有的结论如近交衰退在维持混合交配系统进化中的 主导作用、在濒危物种中保护远缘居群利用远交方 法来提高后代生存力等也受到严重挑战。植物繁育 系统实际上已成为一个以生殖”为核心, 以探讨物 种多样性发生历史、维持机理和保护策略为最终目 的的综合交叉研究。关于植物繁育系统研究报道和 综述自达尔文以来的 140 多年里积累了极其丰富的 资料和文献, 尤其是由于近 20 年传粉生态学受到的 再次重视，几乎翻开任何一期有关植物进化和生态 学的杂志均能找到有关这方面的研究报道, 而且逐 年上升(黄双全等, 2000)。国内最近也发表了许多 相关领域的综述(郭友好, 1994; 周世良等, 1998; 王 崇云等, 1999 ; 黄双全等, 2000; 张大勇等, 2001; 何田 华等，2001）这些学者都分别介绍了交配系统和传 粉生物学中的术语、概念、模型以及部分研究现状, 这些综述对我国从事繁育系统实验和观察的研究者 有着极为重要的参考价值。我们这里主要就繁育系 统学研究中近 5 年内最为活跃的领域，如传粉机制 的多样性、繁育系统多样性的系统发育检验、近交衰 退、混合交配系统的优势以及繁育系统在植物多样 性保护中的作用选择了少量典型的最新文献, 侧重 于繁育系统的野外观察和实验研究进展, 做一较为 全面的总结, 并对今后的发展趋势进行了概括, 以期 能为我国研究工作者提供前沿研究领域的动态信 息。

\section{1 繁育系统中传粉机制的多样性}

异交是形成繁育系统中传粉多样性的主要动 力。异交的优势、有利性和多样性早在 18、19 世纪 就得到了形象而深刻的认识 (Sprengel，1793; Knight , 1799 ; Darwin ,1859；1862；1876)。异交传粉 机制的多样性来自花结构和传粉媒介的相互适应, 其多样性表现在花与传粉媒介两个方面。根据传粉 媒介的不同, 异交又可主要分为:风媒、水媒和虫媒 (主要是指昆虫) 繁育系统。前二者花的构造相对简 
单, 被子植物传粉机制的极大多样性主要表现在动 物传粉的繁育系统中 (Barrett \& Harder ,1996) ,尽管 这三者在很多生态系统或者植物单系发生群中都有 相互密切的联系。花形态 (传粉前机制)方面的适应 机制有雌雄异熟 ( Dichogamy) 、花柱对生 ( Enantiostyly)、异型花 (Herkogamy)、花柱异长 (Heterostyly);生理 (传粉后机制)方面的自交不亲和机制( Self-incompatibility, SI) 与配子体选择有关 (Richards ,1997)。生理 机制隐性的自交不亲和 (Cryptic self-incompatibility， CSI) 机制更是一种巧妙的保证生殖成功的适应机 制:当异花花粉大量存在时,CSI 最大限度地降低同 株花花粉的生殖率, 而异花花粉较少时, 便最大限度 地提高同株花花粉的生殖率。传粉后的每一个环节 都可能是 CSI 选择的对象，如在三型花柱植物 $D e-$ codon verticillatus 中 CSI 是通过调节花粉萌发率的差 异, 如改变花粉管生长速率、胚珠识别选择等来实现 的(Echert \& Allen ,1997)。以 Brassica 为模型研究植 物的自交不亲和分子机理中发现, 柱头和花粉自交 不亲和主要是由 $S$ 位点的 $S R K 、 S P 11$ 和 $S L G$ 基因控 制，它们分别编码柱头和花粉表面相互识别的酶蛋 白来达到接受异花花粉，拒绝自花花粉的目的 ( McCubbin \& Kao 2000)。

毫无疑问，花冠形态特征的性表达多样性是造 成传粉表达形式高度多样性的主要原因, 如为大家 所熟悉的兰科植物中的各种假交媾传粉 (Proctor et $a l ， 1996$ )。花性表达的另一重要组成部分是柱头。 到目前为止,自然界花柱异长有 4 类:两型花柱 (Distyly)、三型花柱 ( Tristyly)、柱头高度两型性 (Stigma-height dimorphism) 和花柱对生 (Enantiostyly, 有异 长雄芯存在), 它们都是通过提高植物个体间花粉散 布的精确性来增加雄性交配效率 (Barrett et al. , 2000)。可变花柱 (Flexistyly) 是花形态对异花传粉的 另一适应机制。李庆军等 (2001) 和 $\mathrm{Li}$ 等 (2001; 2002) ) 在单子叶植物姜科山姜属 (Alpinia) 中发现这 一机制，每个种的花在同一特定时间内都有两种花 柱表现型(李庆军等,2001;Li et al.,2001; 2002)： 柱头上举型 [ Hyperflexistyle, 后又发表文章改为 Anaflexistyle ( $\mathrm{Li}$ et $a$ l. , 2002)] 和柱头下垂型 (Cataflexistyle); 在不同的时间内, 两类花通过花柱位 置的变化来实现花雌、雄性的转变, 同一表现型花的 性表达状态严格一致,避免了同种表现型花间的基 因交流，促进两种表现型间的杂交。前面几种形态 上的适应机制都是雌雄性器官在时间或空间上适 应, 而可变花柱则是时间和空间上适应的共同组合
（Li et al，，2001)。这种雌雄异熟和异型花相结合 的繁育系统在被子植物 9 个目、11 个科和 17 个属中 存在, 被称为雌雄异型异熟 (Heterodichogamy); 价进 化上讲，雌雄异型异熟源自于同步雌雄异熟 ( Synchronous dichogamy) 将向雌雄异株进化, 这个假说还 有待于从历史发生角度的系统发育分析来证明 (Renner, 2001)。李庆军等的研究结果将促使更多 的生物学家去关注植物的暂时性行为的二型性 （Renner，2001），也表明了解花性表达与异花传粉的 多样性仍是当前植物繁育系统研究的重要任务之

以前的研究对花各性器官寿命的多样性未给予 应有的重视, 原因是花寿命的问题在繁育系统的总 结性专著中被忽略 (Primack，1985)。花寿命是指花 开放和有功能的那段持续期 (Primack , 1985 ; Schoen \& Ashman , 1995 ; Ashman \& Schoen , 1994)。这一时 期对理解传粉生态学的动态过程非常重要 (Primack，1985), 因为它决定了花粉和柱头的展现 (Presentation) 时间，而且花寿命延长是对访花频率 低的适应 (Ashman \& Schoen , 1994 ; Bingham \& Orthner , 1998)。花寿命更多的是对环境因子(如温度、湿 度)的适应，而和物种的历史发育关系、传粉者种类 无关 (Primack，1985)，且受繁育系统(自交或者异 交) 影响很大 (Primack, 1985 ;Sato , 2002)。Richardson 和 Stephenson (1989) 发现花粉移取率高的花比花 粉移取率低的花有更短的花寿命，而且雄性持续期 和花中花粉数量也存在相关性 (Devilin \& Stephenson , $1984 ; 1985)$ 。最近的研究发现柱头保持活力与 花粉散布的时间对植物繁育系统的影响不亚于活动 昆虫的种类和频率等因子。长期以来, 人们认为高 山植物由于昆虫种类少、活动频率低, 从而得不到有 效的异花传粉。Bingham 和 Orthner (1998)比较了高 山与低山 Campanula rotundifolia 不同居群影响繁育 系统的各种因素, 发现高山居群长时间的柱头活力 和高效率的传粉效果完全可以抵消由于活动昆虫少 和频率低造成的不利影响。我们对高山龙胆科植物 花对传粉机制的适应机制研究发现，龙胆科植物的 花为适应不同类型的昆虫异花传粉,表现出多种花 形态式样, 并且为适应高原昆虫访花频率低、花寿命 被迫延长而必须在花开放方式上节约能量, 很多种 类的花均表现出有规律的昼夜开放和闭合 (未发表 资料)。除研究花个体的性表达对繁育系统的影响 外，花群体特征在繁育系统中的作用也得到了关注。 花的开放式样 (Floral display) 是某一时间花的大小、 
开放的数量和在空间上的排列模式 (Barrett \& Harder , 1996 ; Barrett , 1998 ; 黄双全等 ,1999,2000;Worley et al.,2000), 是花在群体上的表现 (黄双全等, 1999 2000)。目前研究较多的是花序的开放式样, 如在 Asclepias speciosa 中发现同株异花受精与花序 进化存在相关性 (Finer \& Morgan, 2000)。高山植物 麻花艽 (Gentiana straminea) 的单花表现出有雌雄异 熟和异型花相结合的严格异花传粉机制，但花序却 表现为同株异花受精的方式，从而维持着该植物的 混合交配系统(未发表资料)。

花形态是由传粉者 塑造” 的 (Real, 1983 ; 黄双 全等，2000）这一论断在一定程度上反映了花的多 样性是适应传粉媒介的多样性而引起的。昆虫是被 子植物的主要传粉者, 传粉昆虫涵盖了昆虫的各分 类等级，包括数目众多的种类 (Faegri \& van der Piji, 1979 ;Real ,1983）,这已为大家所熟悉。哺乳动物为 植物传粉是最近才发现的, 首先是在 Protea 灌木中 记载(Wiens \& Rourke,1978), 后来又发现双子叶植 物 Loasaceae 和 Melastomataceae 两个科部分灌木的花 也是由哺乳动物传粉 (Cocucci \& Sérsic,1998), 最新 发现单子叶植物中的来自非洲的一种草本百合 Massonia depressa 也是由 4 种小哺乳动物为之传粉 (Johnson et al. 2001)。鸟类为部分热带植物传粉, 早在达尔文时代就已经被揭示, 但以往鸟类的传粉 主要是通过鸟喙、鸟羽和鸟足等外部可见部位来完 成。萝摩科的 Microloma 的花非常小、花管短、花冠 开口非常小 根据花冠综合特征, 被认为是由昆虫传 粉。但最近 Pauw(1998)发现 Microloma sagittatum 却 是依靠鸟的舌头来进行花粉的传播和异花受精的完 成, 他还指出 Microloma 的其它种类以及具有类似花 形态的夢摩科另一分布在东亚的大属 Dischidia 也 可能是由鸟舌来传粉。以往判断由何种动物传粉主 要是依据花的综合特征, 而这些研究结果则表明, 只 依据花的形态特征来推断传粉机制可能得出十分错 误的结论, 传粉机制的准确论断必须依靠野外的实 地观察 (Cocucci \& Sérsic ,1998;Ollerton ,1998)。从前 未知的各种的植物-传粉者之间的机制被不断发现， 植物和其传粉者之间的关系远比人们以前想象的要 复杂或者说更为精彩, Ollerton(1998) 评论这些成就 时指出 将来的某一天当我读到鱼为某种水生植物 传粉时，我也不会感到奇怪”。这些植物与植物传粉 者之间的精巧机制成为植物多样性发生和维持的重 要因素, 保护植物生物多样性实际上更应重视保护 这种相互关系所构成的生态系统。蝙蝠 (Leptonyc- teris curasoae) 和 Agave macroacantha 及仙人掌的共生 关系就是这其中最为典型的例子, Agavaceae 的很多 植物和许多仙人掌依赖晚上访花的蝙蝠传粉, 它们 之间形成一种共生关系; 蝙蝠迁徙时要从由 Agavaceae 和仙人掌植物的花形成的 花蜜廊道” ( Nectarious corridor) 通过;由于蝙蝠本身受生境破坏, 农 业发展和人类捕杀等因素的影响而数目减少, 这样 位于花蜜廊道上的与蝙蝠共生的这个科的 9 种植物 和 30 多种仙人掌植物就因存在传粉问题而使它们 也长期处于危险状态 (Arizaga et al. 2000)。

\section{2 繁育系统多样性发生和转变的系统发育 检验}

花性表达以及繁育系统的多样性常出现在同一 分类群(种、属、族和科)中或者同一分类群的不同地 理分布地域。花性表达主要与花的一些重要性状 (Characters)有关。这些花性状的演化理论不可避免 地涉及到繁育系统特征生态转换 (Ecological shift) 的 假设 (Armbruster \& Baldwin ,1998)。关于花性状的演 化方向以及繁育系统的生态转换过程使用最多的一 个概念是 适应”，因而在 适应”生态特化的过程中， 出现了很多 似乎一致”的假设, 如花性表达总是从 一般到特化 (Specialized)，从两性到单性，传粉系统 从异交到自交, 传粉媒介从虫媒到风媒, 从广泛性昆 虫传粉到专一性昆虫传粉, 花与昆虫的协同进化等 (Thompson,1994)。由于缺乏独立的检验途径，这些 假设的反向性进化、趋同进化极难得到验证, 并且在 各种生态转换假设中总是存在循环论证 (Circularity) (Luckow \& Bruneau, 1997)。例如, 如果不同祖先种 同时占领一个新生境, 同时出现了适应某生态环境 的繁育系统特征，如何验证繁育系统的趋同? 长期 认为的自交是繁育系统进化的末端, 如何从历史发 生 (Historical) 角度予以验证? Barrett (1995) 指出, 检 验繁育系统的进化有两个完全不同又相互补充的方 法: 1) 操作与繁育系统密切相关的花性状, 同数量遗 传学相结合，通过检测自交衰退和性状的基因定位 来阐明繁育系统的进化,2) 通过重建与繁育系统相 关性状的历史系统发育式样来阐明繁育系统的进化 历史。分支系统方法的引进，建立在最简约或者最 大可能性的分支进化过程，从而使得第二种检验繁 育系统的生态转换和进化成为可能 (Luckow \& Bruneau ,1997)。由于形态学分支分析中不可避免地 要用到花的性状，因而在检验繁育系统的分支过程 之前，对花的性表达性状进行了假设，限制了系统发 
育分析的独立检验目的。独立于形态性状之外的分 子证据的大量获得, 使建立在分子证据之上的历史 系统发育分析可以独立检验繁育系统多样性发生和 转换的过程 (Holsinger ,2000)。繁育系统研究和分子 性状的系统发育分析是宏观进化(生态学)和微观进 化 (分子系统发育) 相结合的一个新生长点。在分子 系统树上标明 (Mapping) 花的性表达性状, 可以看出 其进化历程, 还可以清楚显示繁育系统的转换, 为解 释繁育系统转换的生态学原因提供基础 (Armbruster \& Baldwin , 1998 ; Barrett , 1995 ; Well \& Sakai , 1999)。

Schiedea 和 Alsinidendron 的风媒传粉是与花性 表达二型性 (Dimorphism) (Gynodioecy 雌花两性花异 株, subdioecy 亚雌雄异株和 dioecy 雌雄异株) 的进 化密切相关的, 每次二型性的发生都伴随着繁育系 统向风媒的转换 (Weller et al.,1998)。Johanne 和 Liston (2000) 利用 ITS 序列构建了 Thalictrum 两性 花、雌雄异株和雌花两性花同株各种繁育系统的历 史系统发育关系, 初步结果显示该属中风媒传粉先 于雌雄异株和雌花两性花同株的分化。对海生被子 植物的繁育系统和分子系统发育分析表明, 水媒传 粉 (Hydrophily) 发生了多次, 雌雄单独的性表达状态 (Unisexuality)在一些进化发育支上处于基部,并不 总是演化自水媒（Les et al. , 1997)。长期以来, 人 们均认为适应于昆虫传粉的花结构均是从适应广泛 性昆虫的花向适应特异性昆虫的花进化, 但 Armbruster 和 Baldwin (1998) 在研究大戟科 Dalechampia 各种的传粉生态学的同时, 利用分子数据构建了该 属的历史系统发育关系, 发现传粉模式的进化却是 从特异性传粉种类到非特异性种类。

自交和异交是植物繁育系统研究的一对中心问 题。关于自交和异交之间的相互演化是系统发育检 验的重点。自交与异交相比有两个不可忽视的优 势 :1)生殖成功保障和 2) 传递两套完全相同的基因 (Takebayashi \& Morrell，2001)。“自交是进化的末 端”，主要是因为 1 ) 自交不能作为长期的生殖策略， 自交谱系由于缺乏适应和新物种形成的潜力造成自 交衰退而最终灭绝;2) 自交谱系不能转换为异交 (Takebayashi \& Morrell , 2001)。Goodwillie(1999)在研 究 Lianthus sect. Leptosiphon 9 个种 23 个居群的传粉 特征时，同时利用分子片段( ITS) 构建了这些居群的 历史系统发育关系,他发现在该组的 9 个种从异交 到全部依赖自交的进化至少发生了 3 次。我们对小 檗科足叶草族 (Polophyllum) 具有不同繁育系统的种 类进行了分子系统发育构建, 发现自交在该类群中
不同的地理分布区域: 北美和青藏高原同时出现了 两次 (Liu et al. 2002)。通过对 Amsinckia 的异型花 柱异交 (Heterostylous outcrossers) 和同型花柱自交 (Homostylous selfing)的分子系统重建，支持同型花柱 自交多次来源于异型花柱异交 (Schoen et al. , 1997)。Barrett 等(1997) 利用 $m a t K$ 基因系列构建了 Polemoniaceae 科 77 种具有自交和异交两种繁育系 统的系统发育树，结果发现所有的自交种类都位于 分支树的顶端, 自交来源于异交, 存在多次进化事 件，支持 自交是进化的末端” 的假说。他们还注意 到自交不亲和(Self-incompatibility, SI)机制在该科中 发生了多次,因为具有该机制的 8 个属 (Cobaea， Gilia , Ipomopsis , Loeselia , Linanthus , Leptodactylon , Phlox 和 Polemonium ) 并不是在同一进化支上。我们 在已构建的龙胆科龙胆族獐牙菜亚族分子系统发育 树上( Liu et al. ，2001)，选择部分物种进行了繁育 系统研究 结果发现自交位于几个独立的分支上, 表 明在该类群物种多样化的历史发生过程中, 自交来 源于异交, 存在多次的进化事件发生 [未发表资料]。 最初利用形态和分子资料构建的 Scutellaria angustifolia complex 复合体的系统发育表明自交是祖先状 态来自自交的异交进化事件在该复合体中发生了 3 次 (Olmstead,1989) ,但进一步的外类群选择和性状 分析表明自交来源于异交( Well \& Sakai ,1999;Takebayashi \& Morrell 2001)。尽管细辛属 (Asarum)中具 有吸引昆虫传粉性状的异型花 (Herkogamy) 种类位 于分子系统树上的端部, 而具有自交花性状的种类 位于分支树的基部, 但野外的繁育系统调查发现具 有吸引昆虫传粉性状的异型花却不表现为异交 (Kelly ,1997)。目前还没有研究能确切地证明从严 格的自交演化为异交( Well \& Sakai, 1999 ;Takebayashi \& Morrell, 2001;Holsinger,2000)。异交存在多种机 制, 如雌雄异熟、花柱异长、自交不亲和机制等, 这些 机制可能共同存在于同一进化支上 (如 Polemoniaceae）从而造成异交的趋同或者趋异性进化，自交 可能也存在多种机制, 由于目前对自交的原因研究 较少, 还不能揭示自交机制的多样化。已有的繁育 系统转换研究中对异交和自交的机制未加以区分， 只是简单注明自交或者异交，因而对多次进化事件 只能解释为趋同, 不能具体说明其原因。

利用分子系统研究验证繁育系统的进化, 发现 过去的理论假设在许多类群中确实是正确的, 但在 一些类群中也发现了与传统假设相反的结果, 如在 大戟科 Dalechampia (Armbruster \& Baldwin ,1999)中发 
现特异性传粉并非是不可逆转的, 昆虫并不总是与 花协同进化。这些结果进一步揭示了繁育系统多样 性发生和转换的复杂性, 因而在对繁育系统的生态 原因分析时不应再只是 适应” 和 选择”假说，对自 交衰退、杂交优势以及对极端生态环境适应后的繁 育系统特化失去了适应新环境的潜力等都应视类群 而做相应的评价。

\section{3 近交或自交衰退}

交配系统和居群的遗传结构密切相关, 是影响 群体遗传结构的关键生物学因素之一(葛颂, 1998), 而传粉生物学知识则是解释交配系统特点的关键 (周世良等, 1998)，传粉载体行为是传粉前影响交配 系统的最关键的因素。姊妹种交配系统的分化往往 是物种形成过程中生殖隔离机制之一, 如石荠蓝 (Mosla hangchouensis) 和石香薷 ( M. chinensis) ( 周世 良等,1996), Campanula panclata 和 C. microdonta (Lloyd \& Barrett ,1996) 姊妹种对的分化。交配系统 中研究较多的是近交衰退。近交是自交和血源关系 相近的个体间的交配。自交指自花受精和同株异花 受精, 异交是异株间的交配, 近交不象自交和异交那 样具体, 是相对的概念, 所以每个近交个体近交程度 都是不同的。

近交的有害性表现在造成了个体间遗传多样性 和后代适合度的损失 (Mackenzie et al. ,1999) , 即近 交衰退 (Inbreeding depression)。对自交或者近交种 类的遗传多样性的检测表明，近交确实能造成遗传 多样性的降低, 但遗传多样性的减少是否一定能导 致适应潜力的丧失, 最终造成自交居群的灭绝仍需 要进一步研究 (Takebayashi \& Morrell 2001)。无论如 何，已有的实验证据表明近交衰退确实严重影响了 交配系统的进化和小居群的生存 (Byers \& Waller , 1999)。近交衰退对后代适合度的影响体现在生活 史的各个阶段, 如导致 Sarracenia flava (Sarraceniaceae) 种子数量和质量的下降, 自交的种子产生的后 代在发芽、存活、生长等阶段都不及杂交后代 (Philip \& David 2000)。在 Lupinus arboreus 中近交衰退表现 为结实率有差别, 而发芽、存活、生长等阶段没有明 显差别 (Pamela \& John, 2000)。Verbascum thapsus 生 长发育早期阶段不表现近交衰退 (Donnelly et al. , 1998), 在 Gentianalla germanica 中近交衰退严重导致 结实率降低 ( Fischer \& Matthies ,1997), 在 Hibiscus grandiflorus 和 H. moscheutos (Malvaceae)中近交衰退 都表现在结实过程中, 少数表现在种子萌发阶段, 更
多表现在后代生长方面 (Klips, 2000)。在 Silene virginica 中, 近交衰退的影响不表现在结实时期, 也主 要是表现在后代生长方面 (Dudash \& Fenster, 2001)。 近交衰退的影响和环境相关, 野外环境的近交衰退 比室内更严重 (Charlesworth \& Charlesworth, 1987; Dudash，1990），也和染色体的倍性有关，二倍体比四 倍体的近交衰退严重 (Husband \& Schemske，1997)。 近交衰退和繁育系统密切相关, Husband 和 Schemske (1996)认为各个生活史阶段积累的近交衰退和居群 的自交率呈显著负相关，而且繁育系统明显不同的 物种近交衰退对其影响的阶段也不同,自交占优的 物种这种影响主要表现在种子萌发、后代生长阶段， 而杂交占优的物种却通常表现在较早的种子结实方 面 (Barrett \& Harder ,1996 ;Dudash \& Fenster , 2001)。

近交衰退通常被解释为近交后代增加了表达隐 性有害基因的可能性 (部分显性模型, Partial dominant model), 减少了杂合子的表达利益 (超显性模 型，Overdominant model) (Charlesworth \& Charlesworth , 1987)。部分显性模型预言在有规律的近交居群中 近交衰退影响应受到限制，因为自然选择清除了导 致近交衰退的有害基因, 这一模型初步得到证明 (Husband \& Schemske, 1997)。Klips(2000)发现近交 衰退对 Hibiscus species 的影响是可变的, 南边的居 群严重些, 而靠北边的则受影响小, 从而推测是北边 居群冲破遗传瓶颈，消除了有害基因冰期后重建的 结果。在 Agrostis tenuis 和 Anthoxanthum odoratum 矿 山居群 (Antonovics, 1968)的形成过程中, 也可能经历 了自交居群对自交衰退影响的清除这段艰难的瓶颈 (王崇云等,1999)。自然选择对有害基因的清除 (Purging)程度依赖一些居群和遗传的因素 (Byers \& Waller,1999），生活史早期表达的隐性致死基因能被 近交所清除,而晚期表达的则比较困难 (Husband \& Schemske, 1996)。

近交在交配系统的进化中有着重要的地位, 自 交发生的进化假说目前有好多种 (Wyatt, 1983 ; 王崇 云等, 1999) , 但其功能主要有两点 ( Takebayashi \& Morrell ,2001) : 繁殖保障 ( Baker's law) ( Baker ,1955) 和自动选择优势 (Fisher's model) (Fisher ,1941)。生 态隔离的邻近居群间,自交具有直接的进化意义： Agrostis tenuis 和 Anthoxanthum odoratum 的矿山居群 和草场居群及对重金属的耐受居群和非耐受居群的 对比研究发现, 耐受居群的自花受粉要高于非耐受 居群, 计算机模拟研究也表明自交这种繁殖保障策 略在矿山居群形成初期非常重要 (Antonovics ,1968)。 
自交的众多好处, 也主要来自繁殖保障功能。物种 Clarkia xantiana ssp. xantiana (Onagraceae) 和其亚种 C. xantiana ssp. parviflora 地理分布和交配系统的 差异为证明繁殖保障假说提供了一个理想的系统 (Fausto et al. 2001)。但自动选择优势假说很少有 证明, 而且自交的进化是由于自动选择优势还是繁 殖保障或者二者兼而有之还很难阐明。显然, 这些 假说都需要更多的实验证据来证明。

\section{4 混和交配系统}

近交衰退使近交的便利大打折扣，而远交的代 价很高, 受外界条件影响也大。进化是折中的, 权衡 近交和远交利弊的途径是混和的交配系统。近交使 家系的个体基因型更加专一，家系间出现遗传分化； 远交降低杂合子的一致性，避免群体出现遗传分化。 自然界绝对自交或杂交的类群很少, 大多是二者兼 而有之的混和交配模式(葛颂,1998)。

交配系统是动态的。一方面，它随时间的改变 而变化“, 自然界讨厌永恒的自花受精” ( Sprengel, 1793 ; Knight , 1799 ; Darwin , 1876 ; Proctor et al. , 1996)便说明了交配系统的时间可变性，姊妹种石荠 芳和石香薷的交配系统的分化(周世良等,1996)也 是这种交配系统大时间尺度上变化的例子。小时间 尺度上的变化主要是由群体遗传结构的空间模式和 传粉媒介行为的不稳定造成的。另一方面, 交配系 统也随空间而变化，如分布在 6 个岛上的 Campanula microdonta 居群的杂交率差异极大 (Lloyd \& Barrett 1996)。交配系统的时空动态基本上是由遗传 控制的花的特征决定的, 如花粉/胚珠值、花形态、花 粉大小、自交亲和的程度等 (Lloyd \& Barrett ,1996)。 环境因子也起很大作用，如传粉者的服务水平、自交 和异交花粉到达柱头的时间以及花粉量的大小和组 成等(Wyatt ,1992)。即使在同一个居群中, 也存在早 花和晚花结实率的差异 (Monica et al，2000) 地理 分布不同的居群其自交水平也各不相同, 如居群边 缘自交率高(Wyatt，1983)。

远交的植物本身对雄性投资加大，如花粉/胚珠 值增大及增加吸引昆虫机制维持的能量等。远交还 受环境影响比较大, 高山上昆虫多样性低, 传粉昆虫 少, 很多植物交配系统有向自交方向进化的趋势 (Escaravage et al.,2001)。水生植物黄花萄 ( Limnocharis flava) 也有类似的进化趋势 (宋志平等， 2000）。岛屿和大陆昆虫区系不同，植物的交配系统 也由此发生分化 (Lloyd \& Barrett ,1996)。所以混和
交配系统是对植物本身和环境条件适应的一种折中 的机制。在生境入侵、不可靠的传粉条件，居群的局 部适应等特定的生态条件下，自交是有利的，而且自 交个体向自己的后代传递两个拷贝的基因，杂交只 一个 相比之下自交植物便有自动传递的利益 (Fisher ,1941)。Ipomoea purpurea 杂交率为 60\% 80\%, 花药柱头间的距离 (Anther stigma distance, ASD) 是影 响自交率的主要因素,ASD 越小,自交率越高; 但小 ASD 个体并没有得以稳定进化, 显然选择压力的存 在阻止了有利于自交的基因型的固定从而长期维持 混和的交配系统 (Chang \& Rausher ,1998)。理论研究 表明，近交衰退是阻止自交进化的选择压力 (Lande

\& Schemske , 1985 ; Charlesworth \& Charlesworth , 1987 ; Holsinger , 1991)。Schemske 和 Lande (1985) 认为近 交衰退水平的临界值为 0.5 , 低于 0.5 导致自交传递 即向自交稳定方向进化，高于 0.5 则向杂交稳定方 向进化，只有 0.5 的近交衰退水平才使混和交配系 统得以保持。但 Ipomoea purpurea 的近交衰退水平 小于 0.25 , Chang 等 (1999) 因此指出近交衰退不是 唯一的保持机制 雌、雄适合度组成的近交衰退结合 效应维持了该种植物进化稳定的混和交配系统。基 因的自动传递利益被认为是自交进化的选择压力 （Fisher，1941）,但自花受粉，包括花内自交和同株异 花受精( Intra- and inter-flower self-pollination)，对雄性 适合度有很大影响,这种影响被称为花粉折扣 (Pollen discounting, 翻译和张大勇等 (2001) 有异, 以 供商榷)，即因自花传粉而产生的杂交生殖成功降低 (Barrett，1998; 张大勇等,2001)。花粉折扣对植物 生殖有普遍的影响，主要包括两个方面 (Harder \& Barrett，1995）:1)花粉折扣影响自花受精的进化, 没 有花粉折扣，自交便有自动传递的适合度利益，而花 粉折扣降低了这种利益，从而限制了自交的进化，2) 花粉折扣能促进权衡大花序吸引昆虫之利和同株异 花受精花粉折扣之弊机制的进化, 如传粉后花变化 的进化 (Weiss , 1991；1995；Oberrath \& BohningGaese，1999)。花粉折扣对生殖的影响和自花传粉 的模式有关, 在闭花受精和同株异花受精中影响强 度最大,而在延迟自交中则没有影响（Barrett＆ Harder，1996）花粉折扣的影响还依赖于植株花形 态特征和生态背景 (Fishman，2000)。实验也证明， 花粉折扣在维持 Ipomoea purpurea 混合交配系统中 的作用也十分重要(Chang \& Rausher ,1998)。混合交 配系统的维持机制和选择压力主要是近交衰退、花 粉折扣和雌雄资源分配对策 (Barrett, 1998 ; 张大勇 
等 ,2001) 这些结果还需更多的经验研究来支持, 而 且这种对雄性适合度, 如花粉折扣的关注也是传粉 和交配系统研究从分离走向联合的结果(张大勇等, 2001)。

\section{5 繁育系统研究在植物多样性研究中的作 用}

繁育系统研究是探讨花适应性的桥梁。过度的 近交或远交都可能导致适合度的下降，很显然中间 程度的杂交便成了最适交配。这是 1881 年 Muller 通过 Abutilon 的传粉实验得出来的结论, 即 Muller 法则 (Eugene \& John ,1983)。Waser ( 1983 ) 在 Delphinium nelsonii 和 Ipomopsis aggregata 的野外人工杂 交实验结果支持了这个法则, 还发现实际的杂交距 离通常比最适交配距离近, 所以传粉者和花之间的 关系更多地表现出一种冲突 (Price \& Waser，1979)， 至少在实验的两个种中是这样。这对适应和协同进 化的观点提出了挑战。Herrera 和 Wilson 等 (Lloyd \& Barrett ,1996)对传粉者所施加的选择压力给植物 花变异提供了一个普遍解释的假说提出质疑: 传粉 者是如何驱动花形态的多样化的? 他们并不是否定 “传粉者塑造花形态”, 而是指出研究传粉者与花形 态之间的相互适应过程 (Process)——即交配系统的 形成、稳定和进化趋势, 才能真实揭示花适应性的原 因和机制。最近，对传统认为的花形态上避免自交 的机制，如雌雄异熟、异型花、花柱异长等，从另一个 角度提出解释: 他们也是避免两性冲突 (Sexual interferences ,Barrett，1998，2002)。这种解释存在的理由 很简单，如花柱异长被认为是促进杂交的机制，但花 柱异长也存在于自交不亲和的类群中, 因此明显有 异于原有仅解释为避免自交的观点。自然界中 $72 \%$ 的花性表达状态是两性花（Real，1983; Richards , 1997 ; 黄双全等,2000)，两性花个体既接 受花粉又散布花粉, 最后又用作后代个体的父母本， 在传粉和交配过程中，双重的性角色导致父母本功 能的冲突和折衷 (Barrett, 2002)。这种解释独立于 近交衰退的观点, 也提醒人们不要只从雌性适合度 如近交衰退方面，而且还应该从雄性适合度方面，如 花粉折扣来探讨交配系统进化的选择压力和维持机 制(张大勇等 2001)。

交配系统研究对物种形成机制的研究有重要启 示。交配系统尤其是杂交障碍是物种形成的必要条 件(葛颂，2000)。杂交衰退 (Outcrossing depression)指 父母本基因型的相异性超过一定限度而使后代的适
合度最终下降的现象 ( Eugene \& John ,1983 ;Lynch ， 1991 ;Waser \& Price ,1994; Waser et al. 2000)。Waser 等(Eugene \& John ,1983) 在 Delphinium nelsonii 中的研 究表明最佳传粉距离为 $3 \sim 10 \mathrm{~m}$, 而 Ipomopsis aggregata 为 $1 \sim 10 \mathrm{~m}$, 超过 $10 \mathrm{~m}$ 有明显的杂交衰退。对 杂交衰退的解释有两种假说: 从环境考虑为对局部 环境条件适应的失调（Waser \& Price，1994），从生理 考虑为基因共适应的破坏 (Lynch,1991)。这两种机 制不是排斥的 基因的共适应是对局部环境适应的 基础(Waser et al. 2000)。不管是哪种机制, 杂交衰 退代表一种杂交障碍。物种形成的关键在于生殖隔 离(葛颂，1998），而杂交障碍是一种合子后的生殖隔 离机制,Waser 等(2000)通过对杂交衰退的 3 次研究 发现杂交衰退是可变的, 这种变化是亲代基因和环 境及二者相互作用历史不同的反映。这种可变性的 研究是杂交衰退时空动态研究的一部分。在一定尺 度上杂交障碍的强度对揭示物种形成过程中生殖隔 离有重要意义(Coyne \& Orr ,1997)。研究表明,花粉 来源和量的不同, 花粉的表现(花粉萌发, 穿入柱头 的能力及花粉管生长速率)也就不同 花粉表现和后 代体质均受花粉竞争环境 (花粉来源多样性和花粉 量大小)的影响(Richard,1999)。花粉竞争进而排斥 常常是同域物种形成的主要生殖隔离机制,同域物 种 Hibiscus moscheutos 和 H. laevis 纯花粉杂交可产生 充足的种子, 而这两个种的均匀混合花粉 (50\%) $50 \%$ ) 杂交则各自只有 $8.0 \%$ 和 $7.4 \%$ 的结实率 (Klips, 1999) ;同域种 Helianthus annuus 和 H. petiolaris 中混合花粉杂交结实率不超过 4\% (Rieseberg et $a l ., 1995)$; 在 Iris fulva 和 I. hexagon 中混合花粉杂 交, 前者杂交种子一点都没有, 而后者结实率仅为 27\% (Carney et al. ,1994)。自然状况下由于传粉昆 虫的不专一性, 植物接受的主要是混合花粉从而导 致杂交结实率降低最后导致生殖隔离 (Carney et al. 1994)。总之, 交配系统的分化是物种形成的重 要遗传机制之一(周世良等, 1996 㵧颂, 2000)。

交配系统研究对濒危物种保护具有指导意义。 生物多样性保护的关键之一是保护物种, 而物种濒 危其实就是该种生活史中的一个或几个环节受内外 因素的影响出现危机而使生物个体数量下降的现 象, 至于哪些环节及为何出现危机则涉及到濒危机 制的研究。一些濒危物种的共同特点是人为活动的 影响造成生境破碎化，使物种以小居群的形式存在， 近交便成了不可避免的问题, 近交群体中由于遗传 漂变积累的影响, 群体间遗传分化和群体内遗传一 
致性增强, 而遗传一致性大的居群对可变环境的适 应能力差, 所以近交衰退便成了物种保护和保护区 管理中主要考虑的问题。近交衰退普遍降低了后代 的适合度, 但它的重要性和具体影响是高度可变的 (Philip 2000) ,环境胁迫愈严重近交衰退程度愈高 (Dudash，1990)。不同的交配系统对近交衰退的忍 耐力是不同的, 近交占优的种对近交衰退的忍耐能 力强, 杂交占优的种则弱 (Husband \& Schemske, 1996)。与近交衰退相对, 远交衰退对后代的影响则 很少受关注。交配系统的研究不但对物种濒危机制 的揭示有重要价值, 还对具体保护方法的选择有指 导作用, 甚至可以优化我们的保护方案。Gentianella germinica 是中欧的一种两年生的濒危物种, 实验发 现这个种存在严重的近交衰退, 10 m 处的基因流对 后代适合度有较大贡献, 但令人吃惊的是远距离杂 交实验却表明该种植物也存在明显的远交衰退 (Fischer \& Matthies ,1997)。生境破坏和片段化造成 了生殖的传粉限制和近交程度的增强, 对有这种遗 传效应的物种实现保护的措施之一便是人工基因流 (Artificial gene flow) 的方法 (Oostermeijer et al. , 1995）,但实施之前对交配系统的了解是必不可少 的，因为在近交和远交衰退都存在的种中，如 Gentianella germinica 保护措施如果受杂交优势思想的 影响而一味地用远交来提高后代的生存能力同样是 不科学的。在濒危物种繁育系统研究中对濒危原因 作生态学和遗传学的区分也是十分必要的, 这种区 分有助于采取正确的保护策略。濒危物种 Gentiana pneumonanthe 和 Gentianella uliginosa 都受到生境片断 化的影响，但由于前者实现生殖成功对传粉媒介有 极端依赖性且近交衰退影响较大, 因而保护措施中 要考虑传粉媒介和居群数量, 更重要的是要加强居 群间的基因交流 (Oostermeijer, 1996);而后者 Gentianella uliginosa 是自交的繁育系统，它的保护对策 应更多地考虑生态因素, 如人为活动、牧草收割等 (Petanidou et al. 1998)。

\section{6 繁育系统研究中需要注意的问题和未来 的发展趋势}

描述各种植物种类的繁育系统仍是目前理解植 物繁育系统多样性的首要任务。解释所观察的繁育 系统现象时通常使用最多的概念是“适应” (Richards ,1997)。用 适应” 来解释繁育系统中的观 察现象时要充分理解 适应” 的内涵和外延。适应是 生物学的中心概念, 同时也是引起巨大争论的概念
(Willmer et al. 2000)。适应是生物特有的, 不同的 研究者有不同的定义, 研究的层次不同, 适应的标准 也各异(张昀,1999)。适应被定义为一个缓慢的、不 可逆的过程, 它通过自然选择来调整编码影响后代 适合度的特征的基因频率(Willmer et al，2000)。在 适应的过程中 花特征适应性的解释常常局限于描 述, 并且关键的解释是比较模糊的 (Waser , 1983) 花 特征通过影响传粉过程来影响植物生殖成功, 对这 个过程的评价是必要的 (Campbell et al . , 1991) , 这 种评价有利于揭示花特征的适应性选择和进化的机 制(Campbell et al ., 1996)。研究花特征的适应性和 进化机制有两种方法可循: 1) 自然居群中花表型特 征和后代适合度的相关和回归 (Campbell et al. , 1991 ; Irwin，2000）;2) 通过人工控制实验确定该特 征对传粉效率和生殖成功的影响（Waser，1983; Nillson , 1988 ; Campbell et al . , 1996 ;Kudoh \& Whigham , 1998 ; Fukuda et al. ,2001)。我们认为较好的 方法是把人工控制实验研究和自然研究结合起来: 1) 在自然居群中找出特征差异，做初步的假设，2)通 过人工控制的单因子实验证明假设。通过对比研究 取得的结果才更为可靠。在设计单因子实验时, 还 应考虑统计学上的试验设计思路以减小实验误差 (Ohashi \& Yahara , 1998)。

对植物混合交配系统的研究目前大多是在同一 物种的不同居群内进行的, 这些研究结果除阐明了 植物维持混合交配系统的机制和地理分布模式之 外，还发现了种内居群在交配系统方面的进化与转 换模式(Holsinger ,2000)。今后的繁育系统研究中应 更多的注意种以上水平和群落水平的繁育系统的动 态研究。Ushimaru 和 Kikuzawa(1999)研究了 Calystegia 4 种植物的繁育系统, 发现片段化生境中具有自 交亲合和吸引传粉者特征的种有较大的生殖力。 Mani 和 Saravanan (1999) 对菊科进行了 30 多年的研 究, 发现传粉生态是头状花序进化的导向因子, 为蜜 蜂传粉所塑造的头状花序有被蝴蝶传粉所替代的趋 势。对某一特定生态系统下四季中植物繁育系统和 传粉规律的季节变化 ( Navarro, 2000) 以及在某种生 态因素 (如全球变化) 影响下的繁育系统变化趋势 (Totland,1999)的揭示, 是今后植物繁育系统研究的 另一重点。记载昆虫生活规律的年周期, 观察各种 植物繁育系统的季节变化规律, 揭示群落水平上的 繁育系统中各种植物与昆虫的相互关系, 比只研究 一个群落中某个种的繁育系统关系更能阐明繁育系 统中生物之间的协同进化及其互动适应的生态复杂 
性。

在交配系统、物种分化以及物种保护生物学的 繁育系统研究中, 分子生物学技术起了非常重要的 作用 基本上成了这些领域研究繁育系统必做的内 容, 如估计杂交率、计算遗传多样性分布和空间分布 式样等。今后这方面的工作将继续与物种发生和维 持的时、空相联系, 可能更加侧重于验证已有的模 型, 还将在繁育系统研究的实际应用, 如濒危物种保 护策略、种质资源的利用等方面起到更为重要的作 用。

由于分子遗传和分子生理在探讨繁育系统微观 机理方面的深入发展, 我们理解了繁育系统中一些 长期存在的问题: 如自交不亲和的基因位于什么地 方? 它们又是如何控制自交不亲和过程的 (McCubbin \& Kao 2000 ;Takayama et al. 2001) ? 特别是关于 繁育系统中花结构变异发育的研究, 花发育和分化 的基因模型已经建立和得到验证，转录调控因子 (Transcriptional regulators) 通过作用耙基因 ( Target genes）即结构基因共同产生花形态结构的多样性 (Doebley \& Lukens ,1998)。大尺度上的花结构变异 可能是结构基因变异引起,而近缘种花结构之间的 分化以及种内花表型的变异则可能是由调控基因的 进化产生的 (Doebley \& Lukens, 1998)。但我们必须 看到, 目前很多有关繁育系统分子水平的工作均局 限于一些模式种内 (Savolainen et al. 2000) ,从而对 基因控制繁育系统多样性造成的物种多样性和生态 适应缺乏了解。Clegg 和 Durbin (2000) 研究了 Ipomoea purpurea 植物种内花颜色的控制基因, 并将各 种花颜色性状置于同一个生态环境下进行昆虫传 粉, 通过昆虫的选择检测花颜色性状在分子水平和 生态适应的吻合程度。

分子手段和生态学观察在种以上水平的结合仍 将是今后繁育系统研究的另一重点, 这种研究将进 一步揭示繁育系统分化的生态原因和基因调控位 置 及其相互之间的一致性问题。前面我们介绍了 利用独立于花性表达性状的分子系统树来证实繁育 系统分化的历史原因和生态适应，今后的工作还应 进一步研究单系发生群中不同繁育系统种类在控制 花性表达(如异长花柱、特异性花冠等)性状基因(调 控基因和靶基因) 的进化和控制分化 (Holsinger, 2000) 特别是采用数量性状基因定位方法 (QTL, quantitative trait locus)进行花性表达性状的进一步定 位（Wyatt，1992）,同时要加强研究自交繁育系统的各 种机制和基因定位。研究花表型变异对繁育系统各
方面 (如传粉者种类与行为、交配系统等)的影响或 者反馈, 通过花表型将分子和生态联系起来, 找出它 们在生态过程中生存和适应的关键因子。

\section{参 考 文 献}

Antonovics, J. 1968. Evolution in closely adjacent plant populations. V. Evolution of self-fertility. Heredity, 23: $219 \sim 238$.

Arizaga, S., E. Ezcurra, E. Peters, F. R. de Arellano \& E. Vega. 2000. Pollination ecology of Agave macroacaantha (Agavaceae) in a Mexican tropical desert. II . The role of pollinators. American Journal of Botany, 87: 1011 1017.

Armbruster, W. S. \& B. G. Baldwin. 1998. Switch from specialized to generalized pollination. Nature, 394: 632 .

Ashman, T. L. \& D. J. Schoen. 1994. How long should flowers live? Nature, 371: $788 \sim 791$.

Baker, H.G. 1955. Self-compatibility and establishment after "long distance" dispersal. Evolution, 9: $347 \sim 349$.

Barrett, S. C. H. 1995. Mating-system evolution in flowering plants: micro- and macro-evolutionary approaches. Acta Botanica Netherlands, 44: $385 \sim 402$.

Barrett, S.C.H. 1998. The evolution of mating strategies in flowering plants. Trends in Plant Science, 3: $335 \sim 341$.

Barrett, S. C. H. 2002. Sexual interference of the floral kind. Heredity, 88: $154 \sim 159$.

Barrett, S. C. H. \& L. D. Harder. 1996. Ecology and evolution of plant mating. Trends in Evolution and Ecology, 11: $73 \sim 78$.

Barrett, S. C. H., L. D. Harder \& A. C. Worley. 1997. The comparative biology of pollination and mating in flowering plants. Philosophical Transactions of the Royal Society of London, Series B, 351: $1271 \sim 1280$.

Barrett, S. C. H., L. K. Jesson \& A. M. Baker. 2000. The evolution and function of stylar polymorphisms in flowering plants. Annals of Botany, 85 (Supp. A) : 253 265 .

Bingham, R. A. \& A. R. Orthner. 1998. Efficient pollination of alpine plants. Nature, 391: $238 \sim 239$.

Byers, D. L. \& D. M. Waller. 1999. Do plant populations purging their genetic load? Effects of population size and mating history on inbreeding depression. Annual Review of Ecology and Systematics, 30: $479 \sim 513$.

Carney, S. E., M. B. Cruzan \& M. L. Arnold. 1994. Reproductive interactions between hybridization irises: analysis of pollen tube growth and fertilization success. American Journal of Botany, 81: $1169 \sim 1175$.

Campbell, D. R., N. M. Waser \& M. V. Price. 1991. Components of phenotypic selection: pollen export and flower corolla width in Ipomopsis aggregata. Evolution, 45: 1458 1467.

Campbell, D. R., N. M. Waser \& M. V. Price. 1996. Mechanisms of hummingbird-mediated selection for flower witdth in Ipomopsis aggregata. Ecology, 77: $1463 \sim 1472$.

Chang, S. M., D. Mark \& M. D. Rausher. 1999. The role of inbreeding depression in maintaining the mixed mating system of the common morning glory, Ipomoea purpurea. Evolution, 53: 1366 $\sim 1376$.

Chang, S. M. \& M. D. Rausher. 1998. Frequency-dependent pollen discounting contributes to maintenance of a mixed-mating system in the common morning glory, Ipomoea purpurea. American Naturalist, 152: $671 \sim 683$.

Charlesworth, D. \& B. Charlesworth. 1987. Inbreeding depression and its evolutionary consequences. Annual Review of Ecology and Systematics, 18: $237 \sim 268$.

Chen, X. Y. (陈小勇), N. Li (厉宁) \& L. Shen (沈浪). 2001. The mating system of Ardisia crenata var. bicolor (Myrsinaceae), a subtropical understory shrub in Tiantong National Forest Park, Zhejiang Province. Acta Phytoecologica Sinica (植物 
生态学报), 25: 161 165. (in Chinese with English abstract) Clegg, M. T. \& M. L. Durbin. 2000. Flower color variation: a model for the experimental study of evolution. Proceedings of the National Academy of Sciences, USA, 97: $7016 \sim 7023$.

Cocucci, A. A. \& A. N. Sérsic. 1998. Evidence of rodent pollination in Cajophora coronata (Loasaceae). Plant Systematics and Evolution, 211: $113 \sim 128$.

Coyne, J. A. \& H. A. Orr. 1997. "Patterns of speciation in Drosophila" revisited. Evolution, 51: $295 \sim 303$.

Darwin, C. 1859. On the origins of species. London: Murray.

Darwin, C. 1862. On the various contrivances by which british and foreign orchids are fertilized. Murray: London.

Darwin, C. 1876. The effects of cross and self fertilization in the vegetable kingdom. London: Murray.

Darwin, C. 1877. The different forms of flowers on plants of the same species. London: Murray.

Devilin, B. \& A. G. Stephenson. 1984. Factors that influence the duration of the staminate and pistillate phases of Lobelia cardinalis flowers. Botanical Gazette, 145: $323 \sim 328$.

Devilin, B. \& A. G. Stephenson. 1985. Sex differential floral longevity, nectar secretion, and pollinator foraging in a protandrous species. American Journal of Botany, 72: 303 310 .

Dudash, M. R. 1990. Relative fitness of selfed and outcrossed progeny in self-compatible, protandrous species, Sabatia angularis L. (Gentianaceae) : a comparison in three environments. Evolution, 44: $1129 \sim 1139$.

Dudash, M. R. \& C. B. Fenster. 2001. The role of breeding system and inbreeding depression in the maintenance of an outcrossing mating strategy in Silene virginica (Caryophyllaceae). American Journal of Botany, 88: $1953 \sim 1959$.

Doebley, J. \& L. Lukens. 1998. Transcriptional regulators and the evolution of plant form. Plant Cell, 10: $1075 \sim 1082$.

Dong, M. (董鸣) . 1996. Clonal growth in plants in relation to resource heterogeneity: foraging behavior. Acta Botanica Sinica (植物学报), 38: $828 \sim 835$. (in Chinese with English abstract)

Donnelly, S. E., C. J. Lortie \& L. W. Aarssen. 1998. Pollination in Verbascum thapsus (Scrophulariaceae): the advantage of being tall. American Journal of Botany, 85: $1618 \sim 1625$.

Eckert, G. G. \& M. Allen. 1997. Cryptic self-incompatibility in tristylous Decodon verticillatus (Lythraceae). American Journal of Botany, 84: $1391 \sim 1397$.

Escaravage, N., E. Flubacker, A. Pornon, B. Doche \& I. TillBottraud. 2001. Stamen dimorphism in Rhododendron ferrugineum (Ericaceae) : development and function. American Journal of Botany, 88: $68 \sim 75$.

Eugene, C. J. \& L. R. John. 1983. Handbook of experimental pollination biology. New York: Van Nostrand Reinhold Company.

Faegri, K. \& L. van der Piji. 1979. The principles of pollination ecology. 3rd ed. Oxford: Pergamon Press.

Fausto, J. A., V. M. Eckhart \& M. A. Geber. 2001. Reproductive assurance and the evolutionary ecology of self-pollination in Clarkia xantiana (Onagraceae). American Journal of Botany, 88: $1794 \sim 1800$.

Finer, M. S. \& M. T. Morgan. 2000. Geitonogamy and the evolution of inflorescence design in Asclepias speciosa. American Journal of Botany, 87: 53. (abstract)

Fischer, M. \& D. Matthies. 1997. Mating structure and inbreeding and outbreeding depression in the rare plant Gentianella germanica (Gentianaceae). American Journal of Botany, 84: 1685 1692.

Fisher, R. A. 1941. Average excess and average effect of a gene substitution. Annals of Eugenics, 11: $53 \sim 63$.

Fishman, F. 2000. Pollen discounting and the evolution of selfing in Arenaria uniflora (Caryophyllaceae). Evolution, 54: 1558
1565.

Fukuda, Y., K. Suzuki \& J. Murata. 2001. The function of each sepal in pollinator behavior and effective pollination in Aconitum japonicum var. montanum. Plant Species Biology, 16: 151 157.

Ge, S. (葛颂) . 2000. Advances in plants speciation. In: Li, C. S. (李承森) ed. Advances in plant sciences. Vol. 3. Beijing: China Higher Education Press. 3 15. (in Chinese)

Ge, S. (葛颂) . 1998. Retrospect and prospect for studies of genetic structure in plant populations. In: Li, C. S. (李承森) ed. Advances in plant sciences. Vol. 1. Beijing: China Higher Education Press. $1 \sim 15$. (in Chinese)

Goodwillie, C. 1999. Multiple origins of self-compatibility in Linanthus Section Leptosiphon (Polemoniaceae): phylogenetic evidence from internal-transcribed-spacer sequence data. Evolution, 53: $1387 \sim 1395$.

Guo, Y. H. (郭友好). 1994. Pollination biology and evolutionary botany. In: Chen, J. K. (陈家宽) \& J. Yang (杨继) eds. Plant evolutionary biology. Wuhan: Wuhan University Press. 232 $\sim 280$. (in Chinese)

Harder, L. D. \& S. C. H. Barrett. 1995. Mating cost of large floral displays in hermaphrodite plants. Nature, 373: $512 \sim 515$.

He, T. H. (何田华)\& S. Ge(葛颂). 2001. Mating system, paternity analysis and gene flow in plant population. Acta Phytoecologica Sinica (植物生态学报), 25: $144 \sim 154$. (in Chinese with English abstract)

Holsinger, K. 1991. Inbreeding depression and the evolution of plant mating systems. Trends in Ecology and Evolution, 6: 301 $\sim 308$.

Holsinger, K. E. 2000. Reproductive systems and evolution in vascular plants. Proceedings of the National Academy of Sciences, USA, 97: $7037 \sim 7042$.

Huang, S. Q. (黄双全) \& Y. H. Guo (郭友好). 2000. Advances in the studies of pollination biology. Chinese Science Bulletin(科学通报), 45: 225 237. (in Chinese)

Huang, S. Q. (黄双全)，B． F. Jin (靳宝锋)， Q. F. Wang (主青锋) \& Y. H. Guo(郭友好). 1999. Floral display and pollen flow in a natural population of Sagittaria trifolia. Acta Botanica Sinica(植物学报), 41: $726 \sim 730$. (in Chinese with English abstract)

Husband, B. C. \& D. W. Schemske. 1996. Evolution of magnitude and timing of inbreeding depression in plants. Evolution, 50: $54 \sim 70$.

Husband, B. C. \& D. W. Schemske. 1997. The effect of inbreeding depression in diploid and tetraploid populations of Epilobium angustifolium (Onagraceae): implications for the genetic basis of inbreeding depression. Evolution, 51: $737 \sim 746$.

Irwin, R. E. 2000. Mophorlogical variation and female reproductive success in two sympatric Trillium species: evidence for phenotypic selection in Trillium erectum and Trillium grandiflorum (Liliaceae). American Journal of Botany, 87: $205 \sim 214$.

Johanne, R. \& A. Liston. 2000. Breeding system evolution in the genus Thalictrum (meadow rue) Ranunculaceae. American Journal of Botany, 87: 40. (abstract)

Johnson, S. D. , A. Pauw \& J. Midgley. 2001. Rodent pollination in the African lily Massonia depressa (Hyacinthaceae). American Journal of Botany, 88: $1768 \sim 1773$.

Kelly, L. M. 1997. A cladistic analysis of Asarum (Aristolochiaceae) and implications for the evolution of herkogamy. American Journal of Botany, 84: 1752. (abstract)

Klips, R. A. 1999. Pollen competition as a reproductive isolating mechanism between two sympatric Hibiscus species (Malvaceae). American Journal of Botany, 86: $269 \sim 272$.

Klips, R. A. 2000. Inbreeding depression in variable among populations of rose mallows (Hibiscus species, Malvaceae). American Journal of Botany, 87: 45. (abstract) 
Knight, T. 1799. Experiments on the fecundation of vegetables. Philosophical Transactions of the Royal Society, 89: 195 204.

Kudoh, H. \& D. F. Whigham. 1998. The effect of petal size manipulation on pollinator / seer-predator mediated female reproductive success of Hibiscus moscheutos. Oecologia, 117: $70 \sim 79$.

Lande, R. \& D. W . Schemske. 1985. The evolution of self-fertilization and inbreeding depression in plants: genetic models. Evolution, 39: $24 \sim 40$.

Les, D. H., M. A. Cleland \& M. Waycot. 1997. Phylogenetic studies in Alismatidae. II. Evolution of marine angiosperms (seagrasses) and hydrophyly. Systematic Botany, 22: $443 \sim 463$.

Li, Q. J., Z. F. Xu, W. J. Kress, Y. M. Xia, L. Zhang, X. B. Deng, J. Y. Gao \& Z. L. Bai. 2001. Flexible style that encourages outcrossing. Nature, 410: 432.

Li，Q. J. (李庆军)，Z．F. Xu (许再富)，Y. M. Xia (夏永 梅), L. Zhang (张玲), X. B. Deng (邓晓宝) \& J. Y. Gao (高江云). 2001. Study on the flexistyly pollination mechanism in Alpina plants (Zingiberaceae). Acta Botanica Sinica (植物学 报), 43: $364 \sim 369$. (in Chinese with English abstract)

Li, Q. J., W. J. Kress, Z. F. Xu, Y. M. Xia, L. Zhang, X. B. Deng \& J. Y. Gao. 2002. Mating system and stigmatic behavior during flowering of Alpinia kwangsiensis (Zingiberaceae). Plant Systematics and Evolution, 232: $123 \sim 132$.

Liu, J. Q., Z. D. Chen \& A. M. Lu. 2001. A preliminary study of the phylogeny of the Swertiinae (Gentianaceae), based on ITS data. Israel Journal of Plant Science, 43: $301 \sim 308$.

Liu, J. Q., Z. D. Chen \& A. M. Lu. 2002. Molecular evidence for the sister relationship of the eastern Asia-North American intercontinental species pair in the Podophyllum group (Berberidaceae). Botanical Bulletin of Academia Sinica, 43: $147 \sim 154$.

Lloyd, D. G. \& S. C. H. Barrett. 1996. Floral biology. New York: Chapman \& Hall.

Luckow, M. \& A. Bruneau. 1997. Circularity and independence in phylogenetic tests of ecological hypotheses. Cladistics, 13: $145 \sim 151$.

Lynch, M. 1991. The genetic interpretation of inbreeding depression and outbreeding depression. Evolution, 45: $622 \sim 629$.

Mackenzie, A., A. S. Ball \& S. R. Virdee. 1999. Instant notes in ecology. Beijing: Science Press.

McCubbin, A. G. \& T. H. Kao. 2000. Molecular recognition and response in pollen and pistil interactions. Annual Review of Cell Development Biology, 16: $333 \sim 364$.

Mani, M. S. \& J. M. Saravanan. 1999. Pollination ecology and evolution in Compositae (Asteraceae). New Hampshire: Science Publishers Inc.

Monica, M., G. Pablo \& G. Javier. 2000. Patterns of fruit set within inflorescence of Ancratium maritpium (Amaryllidaceae) : nouniform pollination, resource limitation, or architectural effects? American Journal of Botany, 87: $493 \sim 501$.

Navarro, L. 2000. Pollination ecology of Anthyllis vulneraria subsp. vulgaris (Fabaceae): nectar robbers as pollinators. American Journal of Botany, 87: $980 \sim 985$.

Nillson, L. A. 1988. The evolution of flowers with deep corolla tubes. Nature, 334: $147 \sim 149$.

Oberrath, R. \& K. Bohning-Gaese. 1999. Floral color change and the attraction of insect pollinators in lungwort (Pulmonaria collina). Oecologia, 121: $383 \sim 391$.

Ohashi, K. \& T. Yahara. 1998. Effects of variation in flower number on pollinator visits in Cirsium purpuratum (Asteraceae). American Journal of Botany, 85: $219 \sim 224$.

Ollerton, J. 1998. Sunbird surprise for syndromes. Nature, 394: $726 \sim 727$.

Olmstead, R. 1989. Phylogeny, phenotypic evolution, and biogeography of the Scutellaria angustifolia complex (Lamiaceae) : inference from morphological and molecular data. Systematic Botany, 14: $320 \sim 328$.
Oostermeijer, J. G. B. 1996. Population viability of the rare Gentiana pneumonanthe: the relative importance of demography, genetics and reproductive biology. Ph. D. dissertation of University of Amsterdam, the Netherlands.

Oostermeijer, J. G. B., R. G. M. Altenburg \& H. C. M. Den Nijs. 1995. Effects of outcrossing distance and selfing on fitness components in the rare Gentiana penumonanthe (Gentianaceae). Acta Botanica Netherlands, 44: $257 \sim 268$.

Pamela, M. K. \& L. M. John. 2000. Outcrossing rate and inbreeding depression in the perennial yellow bush lupine, Lupinus arboreus (Fabaceae). American Journal of Botany, 87: 652 660.

Pauw, A. 1998. Pollen transfer on birds' tongues. Nature, 394: $731 \sim 732$.

Petanidou, T., A. C. Ellis-Adam, J. C. M. den Nijs \& J. G. B. Oostermeijer. 1998. Pollination ecology of Gentianella uliginosa, a rare annual of the Dutch coastal dunes. Nordic Journal of Botany, 18: $537 \sim 548$.

Philip, W. H. 2000. Inbreeding depression in conservative biology. Annual Review of Ecology and Systematics, 31: 139 162 .

Philip, M. S. \& N. K. David. 2000. Inbreeding, outbreeding and heterosis in the yellow pitcher plant, Sarracenia flava (Sarraceniaceae) in Virginia. American Journal of Botany, 87: 1628 1633 .

Price, M. V. \& N. M. Waser. 1979. Pollen dispersal and optimal outcrossing in Delphinium nelsoni. Nature, 277: $294 \sim 297$.

Primack, R. B. 1985. Longevity of individual flowers. Annual Review of Ecology and Systematics, 16: $15 \sim 37$.

Proctor, H. C. \& L. D. Harder. 1995. Effect of pollination success on floral longevity in the orchid Calypso bulbosa (Orchidaceae). American Journal of Botany, 82: $1131 \sim 1136$.

Proctor, M. , P. Yeo \& A. Lack. 1996. The natural history of pollination. London: HarperCollins.

Real, L. 1983. Pollination biology. Florida: Academic Press.

Richard, A. N. 1999. The effects of pollen load size and donor diversity on pollen performance, selective abortion, and progeny vigor in Mirabilis jalapa (Nyctaginaceae). American Journal of Botany, 86: $261 \sim 268$.

Richards, A. J. 1997. Plant breeding systems. 2nd ed. London: Chapman \& Hall.

Richardson, T. E. \& A. G. Stephenson. 1989. Pollen removal and pollen deposition affect the duration of the staminate and pistillate phases in Campanula rapunculoides. American Journal of Botany, 76: $532 \sim 538$.

Renner, S. S. 2001. How common is heterodichogamy? Trends in Ecology and Evolution, 16: $595 \sim 597$.

Rieseberg, L. H., A. M. Desrochers \& S. J. Youn. 1995. Interspecific pollen competition as a reproductive barrier between sympatric species of Helianthus (Asteraceae). American Journal of Botany, 82: $551 \sim 559$.

Sato, H. 2002. The role of autonomous self-pollination in floral longevity in varieties of Impatiens hypophylla (Balsaminaceae). American Journal of Botany, 89: $263 \sim 269$.

Savolainen, O., C. H. Langley, B. P. Lazzaro \& H. Freville. 2000. Contrasting patterns of nucleotide polymorphism at the alcohol dehydrogenase locus in the outcrossing Arabidopsis lyrata and the selfing Arabidopsis thaliana. Molecular Biology and Evolution, 17: $645 \sim 655$.

Schemske, D. W. R. \& R. Lande. 1985. The evolution of selffertilization and inbreeding depression in plants. 2. Empirical observations. Evolution, 37: 523 539.

Song, Z. P. (宋志平), Y. H. Guo (郭友好) \& S. Q. Huang (黄双全). 2000. Studies on the breeding system of Limnocharis flava (Butomaceae). Acta Phytotaxonomica Sinica(植物分类学 报), 38: $53 \sim 59$. (in Chinese with English abstract)

Schoen, D. J. \& T. L. Ashman. 1995. The evolution of flower 
longevity: resource allocation to maintenance versus construction of repeated parts in modular organisms. Evolution, 49: 131 139

Schoen, D. J., M. O. Johnston, A. M. L'Heureux \& J. V. Marsolais. 1997. Evolutionary history of the mating system in Amsinckia (Boraginaceae). Evolution, 51: 1090.

Sprengel, C. K. 1793. Das entdeckte Geheimniss der Natur im Bau und in der Befruchtung der Blumen. Berlin: Friedrich Vieweg.

Takayama, S., H. Shimosato, H. Shiba, M. Funato, F. S. Che, M. Watanabe, M. Iwano \& A. Isogai. 2001. Direct ligand-receptor complex interaction controls Brassica self-incompatibility. Nature, 413: $534 \sim 538$.

Takebayashi, N. \& L. P. Morrell. 2001. Is self-fertilization an evolutionary dead end? Revisiting an old hypothesis with genetic theories and a macro-evolutionary approach. American Journal of Botany, 88: $1143 \sim 1150$.

Thompson, J. N. 1994. The coevolutionary process. Chicago: University of Chicago Press.

Totland, O. 1999. Effects of temperature on performance and phenotypic selection on plant traits in alpine Ranunculus acris. Oecologia, 120: $242 \sim 251$.

Ushimaru, A. \& K. Kikuzawa. 1999. Variation of breeding system, floral rewards, and reproductive success in clonal Calystegia species (Convolvulaceae). American Journal of Botany, 86: $436 \sim 446$.

Wang, C. Y. (王崇云) \& C. L. Dang (党承林). 1999. Plant mating system and its evolutionary mechanism in relation to population adaptation. Journal of Wuhan Botanical Research(武汉植 物研究), 17: 163 172. (in Chinese with English abstract)

Wang, X. F. (汪小凡) \& J. K. Chen (陈家宽) . 2001. Floral expression, pollination mechanism and mating system of Sagittaria potamogetifolia. Acta Phytoecologica Sinica (植物生态学 报), 25: $155 \sim 160$. (in Chinese with English abstract)

Waser, N. M. 1983. The adaptive nature of floral traits: ideas and evidence. In: Real, L. ed. Pollination biology. London: Academic Press. $242 \sim 285$.

Waser, N. M. \& M. V. Price. 1994. Crossing distance effects in Delphinium nelsonii: outbreeding and inbreeding depression in progeny fitness. Evolution, 48: $842 \sim 852$.

Waser, N. M., M. V. Price \& R. G. Shaw. 2000. Outbreeding depression varies among cohorts of Ipomopsis aggregata planted in nature. Evolution, 54: $485 \sim 491$.

Weiss, M. R. 1991. Floral color change as cues for pollinators.
Nature, 354: $227 \sim 229$.

Weiss, M. R. 1995. Floral color change: a widespread functional convergence. American Journal of Botany, 82: $167 \sim 185$.

Well, S. G. \& A. K. Sakai. 1999. Using phylogenetic approaches for the analysis of plant breeding system evolution. Annual Review of Ecology and Systematics, 30: $167 \sim 199$.

Weller, S. G., A. K. Sakai, A. E. Rankin, A. Golonka, B. Kutcher \& K. E. Ashby. 1998. Dioecy and the evolution of pollination systems in Schiedea and Alsinidendron (Caryophyllaceae: Alsinoideae) in the Hawaiian Islands. American Journal of Botany, 85: $1377 \sim 1388$.

Wiens, D. \& J. P. Rourke. 1978. Rodent pollination in southern African Protea spp. Nature, 276: 71.

Willmer, P., G. Stone \& I. J. Malden. 2000. Environmental physiology of animal. Oxford: Blackwell Science.

Worley, A. C., A. M. Baker, J. D. Thompson \& S. C. H. Barrett. 2000. Floral display in Narcissus: variation in flower size and number at the species, population, and individual levels. International Journal of Plant Science, 161: 69 79 .

Wyatt, R. 1983. Pollinator-plant interactions and the evolution of breeding systems. In: Real, L. ed. Pollination biology. Orlando: Academy Press. $51 \sim 95$.

Wyatt, R. 1992. Ecology and evolution of plant reproduction. New York: Chapman \& Hall.

Zhang, D. M. (张冬梅), X. H. Shen (沈熙环) \& T. H. He (何田华). 2001. A paternity analysis of seeds from different clones in a Pinus tabulaeformis Carr. seed orchard. Acta Phytoecologica Sinica (植物生态学报), 25: 166 174. (in Chinese with English abstract)

Zhang, D. Y. (张大勇) \& X. H. Jiang(姜新华). 2001. Mating system evolution, resource allocation, and genetic diversity in plants. Acta Phytoecologica Sinica (植物生态学报), 25: 130 143. (in Chinese with English abstract)

Zhang, Y. (张昀). 1999. Evolution of organism. Beijing: Peking University Press. (in Chinese)

Zhou, S. L. (周世良)，K. Y. Pan (潘开玉) \& D. Y. Hong (洪德元). 1996. Comparative studies on pollination biology of Mosla hangchouensis and M. chinensis (Labiatae). Acta Botanica Sinica (植物学报), 37: 530 540. (in Chinese with English abstract)

Zhou, S. L. (周世良) \& D. Y. Hong (洪德元). 1998. Recent advances and trends in pollination biology. In: Li, C. S. (李承 森) ed. Advances in plant sciences. Vol. 1. Beijing: China Higher Education Press. (in Chinese) 